\title{
Republished: Transradial approach in the treatment of a sacral dural arteriovenous fistula: a technical note
}

\author{
Emanuele Orru, ${ }^{\oplus 1}$ Chun On Anderson Tsang, ${ }^{\oplus 2}$ Jesse M Klostranec, ${ }_{1}^{3}$ Vitor M Pereira ${ }^{4}$
}

${ }^{1}$ Department of Interventional Neuroradiology, Toronto Western Hospital, Toronto, Ontario, Canada ${ }^{2}$ Department of Surgery, The University of Hong Kong, Hong Kong, Hong Kong

${ }^{3}$ Division of Neuroradiology, Joint Department of Medical Imaging, Toronto Western Hospital, Toronto, Ontario, Canada

${ }^{4}$ Department of Medical Imaging, Toronto Western Hospital, Toronto, Ontario, Canada

\section{Correspondence to} Dr Emanuele Orru; surgeon. ema@gmail.com

Republished with permission from BMJ Case Reports Published 31 March 2019; doi: 10.1136/bcr-2019-014834

Accepted 22 February 2019 Published Online First 22 May 2019

\section{SUMMARY}

Sacral dural arteriovenous fistulas (SDAVFs) are rare, constituting no more than $10 \%$ of all spinal dural fistulas. They are most commonly fed by the lateral sacral artery (LSA), a branch of the internal iliac artery (IIA). Catheterization of this vessel requires either a crossover at the aortic bifurcation in cases of right femoral access or retrograde catheterization from the ipsilateral common femoral artery. We present the case of a 79-year-old man with tethered cord syndrome and a symptomatic SDAVF fed by two feeders from the left LSA. Spinal diagnostic angiography was made exceptionally challenging by an aorto-bi-iliac endograft, and selective catheterization of the left IIA was not possible. The patient could not undergo surgery due to multiple comorbidities, therefore embolization was considered the best approach. The procedure was carried out through a transradial access (TRA) with Onyx and n-butyl cyanoacrylate. The SDAVF was successfully treated and the patient made a full neurological recovery.

\section{BACKGROUND}

Sacral dural arteriovenous fistulas (SDAVFs) are rare, constituting approximately $10 \%$ of spinal fistulas. ${ }^{1}$ Most often, they arise from dural arterial feeders from the lateral sacral artery (LSA). ${ }^{2}$ If embolization is deemed appropriate, selective cannulation of the internal iliac artery (IIA) with a guiding catheter is necessary in order to reach the fistulous point with a microcatheter. In cases of extremely tortuous aorto-iliac vessels or distorted anatomy secondary to prior endovascular intervention for aortic aneurysms, this manoeuver can be technically difficult and sometimes impossible, hence preventing appropriate treatment of the fistula. Transradial access (TRA) can obviate the need for complex or unfeasible contralateral crossovers or reverse curve catheter manoeuvers and makes catheterization of the IIA relatively straightforward. Recently, TRA has been gaining more popularity in the neuroendovascular field. The present case report offers, to our knowledge, the first report of a spinal vascular malformation treated by TRA and emphasizes the importance of this approach in the neurointerventionalist's armamentarium for cases that would be challenging via transfemoral access, even beyond the cranio-cervical area.

\section{CASE PRESENTATION}

A 79-year-old man with a history of spina bifida and tethered cord, who at baseline had to self-catheterize to urinate and needed a walker to ambulate, presented with slowly progressing bilateral lower extremity weakness that in 10 months rendered him unable to walk. An MRI ordered to evaluate the lumbar spine after a fall suggested the presence of a spinal vascular malformation. His past medical
A

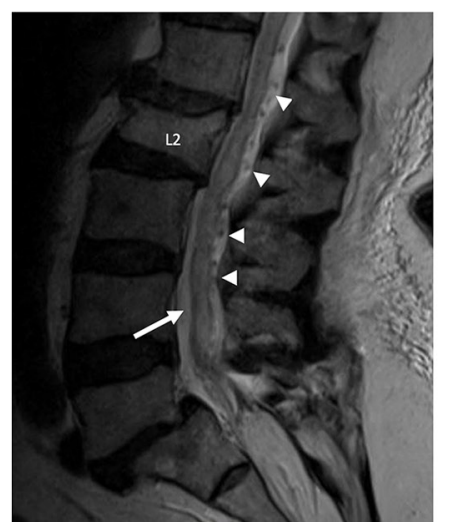

B

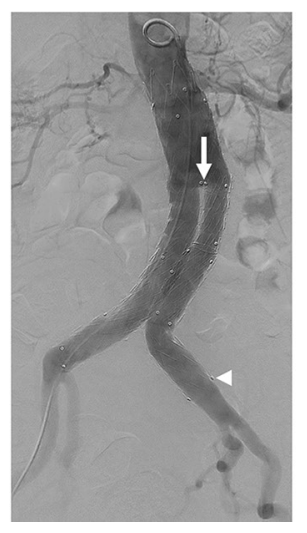

C

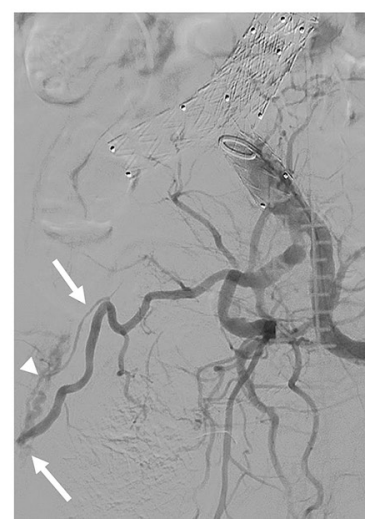

Figure $1 \mathrm{MRI}$ and spinal angiography of a 79-year-old man with a sacral dural arteriovenous fistula (SDAVF). (A) Sagittal T2-weighted MRI of the lumbar spine showing multiple serpiginous flow voids along the posterior surface of the spinal cord (arrowheads). Note the edematous tethered cord (arrow) and an L2 fracture from a recent fall. (B) Pelvic aortogram through a pigtail high-volume injection showing the aorto-bi-iliac graft. The reconstructed iliac bifurcation (arrow) has a 180 degree configuration that did not allow cross-catheterization of the left iliac arteries. Reverse curve catheters could not be reformed due to the edge of the distal end of the left iliac graft (arrowhead). (C) Left common iliac pigtail arteriography demonstrating an SDAVF fed by two feeders from the left lateral sacral artery (arrows). Note the very slow drainage in the enlarged presacral veins directed cranially towards the spinal canal (arrowhead). 
history was also remarkable for a long-standing polyvasculopathy requiring multiple coronary bypass procedures and endovascular repair of an abdominal aortic aneurysm with a covered aortic stent plus bilateral iliac grafts. The patient was $187 \mathrm{~cm}$ tall and had hypertension and obesity, with a body mass index of 43 .

\section{INVESTIGATIONS}

MRI of the lumbar spine (figure 1A) showed an L2 fracture and the known tethered cord. Cord oedema and serpiginous flow voids along the posterior surface of the spinal cord suggested an underlying spinal vascular malformation.

At spinal angiography, an initial pelvic aortogram demonstrated an SDAVF fed by branches of the left LSA at the level of the inferior third of the sacrum. The aorto-bi-iliac endograft made the procedure extremely challenging technically (figure 1B). Cross-catheterization of the left IIA from a right femoral approach and direct cannulation from a left-sided approach were not technically feasible despite attempts with multiple reverse curve catheters. The extreme curve of the IIA prevented stable microcatheterization of the IIA from a non-selective catheter in the external iliac artery. Diagnostic injections eventually carried out via a pigtail catheter in the left common iliac artery showed two radiculomeningeal feeders from the LSA directed to the SDAVF (figure 1C). Venous drainage was relatively slow and directed cranially, reaching the level of the thoracic spinal cord, compatible with the flow voids seen on an MRI performed at an outside institution.

\section{TREATMENT}

Given the suboptimal cardiovascular profile and the tethered cord, it was agreed to proceed with endovascular embolization rather than surgery. Right TRA was chosen in order to have the

A
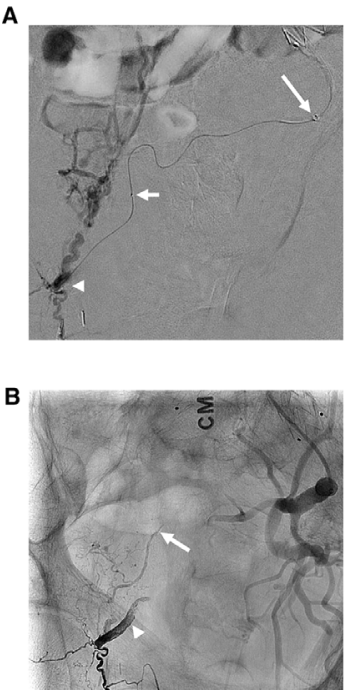

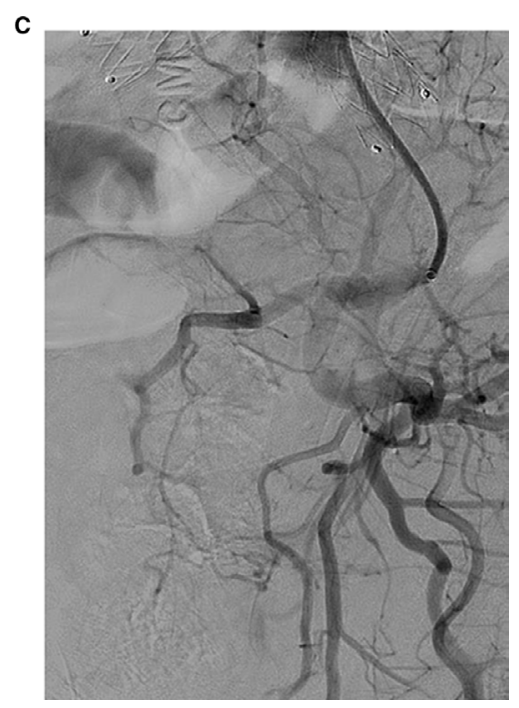

Figure 2 Onyx and glue embolization of the fistula. (A) Superselective arteriogram through a 1.7F Apollo microcatheter at the level of the most distal fistulous point (arrowhead) showing extensive craniallydirected venous drainage in the dilated presacral veins. Note the Sofia catheter lodged in the proximal left internal iliac artery (long arrow) and the proximal marker of the detachable tip of the microcatheter (short arrow). (B) Unsubtracted image from a left internal iliac angiogram shows an Onyx cast filling the most distal fistulous feeder (arrowhead). A slightly less radio-opaque glue cast is seen more superiorly within the embolized proximal feeder to the lesion. (C) Left internal iliac arteriogram demonstrating occlusion of the sacral fistula. straightest path possible for selection of the left IIA with the guiding catheter. A 6F dedicated glidesheath (Terumo, Tokyo, Japan) was introduced under ultrasound guidance into the right radial artery, and a $6 \mathrm{~F} 131 \mathrm{~cm}$ Sofia Plus catheter (Microvention, Aliso Viejo, California, USA) was navigated retrogradely into the left IIA, negotiating the aorto-iliac graft with a 0.038 $180 \mathrm{~cm}$ shapeable hydrophilic wire. A $165 \mathrm{~cm}$ Apollo microcatheter (Medtronic, Friedly, Minnesota, USA) was then advanced over a 0.007 Hybrid microwire (Balt, Montmorency, France) into the LSA until the level of the most distal fistulous point, which was embolized with Onyx 18 (Medtronic) (figure 2A). The second more proximal feeder was selected in the same fashion with a $165 \mathrm{~cm}$ Magic 1.2 microcatheter (Balt) due to significant tortuosity and was embolized with a 2:1 mixture of glue (N-butyl cyanoacrylate) and lipiodol (figure $2 \mathrm{~B}$ ), successfully occluding the fistula (figure $2 \mathrm{C}$ ).

\section{OUTCOME AND FOLLOW-UP}

The patient tolerated the procedure well and slowly improved. At 3 months he ambulates again with a walker.

\section{DISCUSSION}

Similar to their more common thoracic counterparts, SDAVFs can lead to devastating venous hypertensive myelopathy. Diagnosis is made through a complete spinal angiography including a pelvic aortogram and selective injections of the median sacral artery and bilateral external and internal iliac arteries, as these malformations most often arise from the LSA. ${ }^{2}$ Treatment requires positioning of a guiding catheter in the IIA and navigation of a microcatheter as close to the fistula as possible to deliver the embolic agent. In cases with extremely convoluted aorto-iliac anatomies or previously placed stents/grafts, stable transfemoral access to the IIA can become extremely challenging or impossible. In our case, the iliac grafts prevented reformation of the reverse curve of Simmons and Mikaelson catheters. TRA allowed for a relatively straight cannulation of the IIA, and consequently LSA.

TRA has been gaining growing popularity for cervico-cranial neurointerventional procedures, with some institutions using it as their standard approach. ${ }^{45}$ TRA has been described by a some authors for iliac and uterine interventions with excellent clinical results and acceptable fluoroscopic times. ${ }^{6-9}$ Advantages include fewer hemorrhagic complications, notably eliminating the risk for the rare but deadly retroperitoneal hematoma, increased post-procedural comfort and, as in the presented case, access to vessels that would not be reachable through the transfemoral approach. Device size and length are key considerations in TRA. Most radial arteries accommodate $6 \mathrm{~F}$ sheaths, and successful usage of $8 \mathrm{~F}$ systems has been reported for arteries larger than $2.5 \mathrm{~mm}$, although with a potentially higher occlusion risk. ${ }^{10} \mathrm{In}$ the case of distal lesions, the device length can also be a problem as the treatment target might be too distal even for the longest microcatheter. Our patient was taller than average, therefore we preoperatively measured the length of the right arm and abdomen in order to choose catheters long enough to cannulate target vessels without running out of length.

The presented case is, to our knowledge, the first report of an SDAVF-or of a spinal vascular malformation in generaltreated by TRA. This approach, granted appropriate equipment selection, should be in the neurointerventionalist's armamentarium for the treatment of spinovascular pathologies with an otherwise unfeasible aorto-iliac anatomy.

Contributors All authors participated in the procedure and contributed to the 


\section{Learning points}

- Complete spinal angiography must include a pelvic aortogram, median sacral artery, and bilateral iliac injections in order to diagnose a sacral dura arteriovenous fistula.

- Particularly tortuous aorto-iliac anatomy or previous stents can prevent cannulation of the internal iliac artery, jeopardizing the feasibility of endovascular treatment for peripheral neurovascular lesions.

- Transradial access can be a feasible and effective approach to catheterize otherwise unreachable sacral arteries and, potentially, intersegmental arteries.

- In case of transradial access, accurate preoperative choice of device size and length is mandatory, given the distance from the lesion to the access point.

manuscript drafting and review.

Funding The authors have not declared a specific grant for this research from any funding agency in the public, commercial or not-for-profit sectors.

Competing interests None declared.

Patient consent for publication Not required.
Provenance and peer review Not commissioned; externally peer reviewed.

\section{REFERENCES}

1 Gioppo A, Faragò G, Giannitto C, et al. Sacral dural arteriovenous fistulas: a diagnostic and therapeutic challenge: single-centre experience of 13 cases and review of the literature. J Neurointerv Surg 2018;10:415-21.

2 Hong T, Park JE, Ling F, et al. Comparison of 3 different types of spinal arteriovenous shunts below the conus in clinical presentation, radiologic findings, and outcomes. AJNR Am J Neuroradiol 2017;38:403-9.

3 Chen J, Gailloud P. Safety of spinal angiography: complication rate analysis in 302 diagnostic angiograms. Neurology 2011;77:1235-40.

4 Snelling BM, Sur S, Shah SS, et al. Transradial access: lessons learned from cardiology. J Neurointerv Surg 2018;10:487-92.

5 Furtado de Sá BR. Transradial - diagnóstico e interveçao coronária e extracardiaca second. Editora Atheneu: Sao Paulo, Rio de Janeiro, Belo Horizonte, 2009:289-311.

6 Lorenzoni R, Mazzoni A, Lazzari M, et al. Radial artery access for above the knee angioplasty: a feasibility study. Eurolntervention 2011;7:924-9.

7 Resnick NJ, Kim E, Patel RS, et al. Uterine artery embolization using a transradial approach: initial experience and technique. J Vasc Interv Radiol 2014;25:443-7.

8 Shinozaki N, Ogata N, Ikari Y. Initial results of transradial iliac artery stenting. Vasc Endovascular Surg 2014;48:51-4.

9 Mortensen C, Chung J, Liu D, et al. Prospective study on total fluoroscopic time in patients undergoing uterine artery embolization: comparing transradial and transfemoral approaches. Cardiovasc Intervent Radiol 2019;42:441-7.

10 Snelling BM, Sur S, Shah SS, et al. Transradial Approach for Complex Anterior and Posterior Circulation Interventions: Technical Nuances and Feasibility of Using Current Devices. Oper Neurosurg 2018 [Epub ahead of print 28 Nov 2018]. 